\title{
ON COMPLETE HYPERSURFACES OF NONNEGATIVE SECTIONAL CURVATURES AND CONSTANT $m$ TH MEAN CURVATURE ${ }^{1}$ \\ BY \\ PHILIP HARTMAN
}

\begin{abstract}
The main - result is that if $M=M^{n}$ is a complete Riemann manifold of nonnegative sectional curvature and $X: M \rightarrow R^{n+1}$ is an isometric immersion such that $X(M)$ has a positive constant $m$ th mean curvature, then $X(M)$ is the product of a Euclidean space $R^{n-d}$ and a $d$-dimensional sphere, $m<d<n$.
\end{abstract}

1. Introduction. Let $n \geqslant 2, M=M^{n}$ a Riemann manifold, and $X: M \rightarrow$ $R^{n+1}$ an isometric immersion into Euclidean space. Let $\lambda_{1}(x)<\cdots<\lambda_{n}(x)$ be ordered principal curvatures of $X(M)$. In analogy with the case $n=2$, we call $X(M)$ a Weingarten hypersurface (or a $W$-hypersurface) if $W\left(\lambda_{1}(x), \ldots, \lambda_{n}(x)\right) \equiv$ const for some nontrivial function $W(\lambda)=$ $W\left(\lambda_{1}, \ldots, \lambda_{n}\right)$.

Let $M$ be complete and have nonnegative sectional curvatures. Without any assumption that $X(M)$ is a Weingarten hypersurface, a theorem of Sacksteder [15] implies that $M$ and $X$ have factorizations,

$$
M=R^{n-d} \times M_{0}^{d} \text { and } X=X_{1} \times X_{0}
$$

such that $M_{0}^{d}$ is a complete Riemann manifold, the first map $X_{1}$ in

$$
X_{1}: R^{n-d} \rightarrow R^{n-d} \text { and } X_{0}: M_{0}^{d} \rightarrow R^{d+1}
$$

is the identity and the second is an isometric immersion; $X_{0}\left(M_{0}^{d}\right)$ does not contain any (complete) lines and is the boundary of a convex body; cf. [8] for an analogue when $X: M^{n} \rightarrow R^{n+p}$. Of course, either factor $R^{n-d}$ or $M_{0}^{d}$ can be missing (i.e., reduce to a point). If, in addition, it is supposed that $X(M)$ is a $W$-Weingarten hypersurface, then it is of interest to find conditions on $W$ which assure that, in the Sacksteder decomposition (1.1) of $X(M)$,

(a) $X_{0}\left(M_{0}^{d}\right)$ is compact, or even

(b) $X_{0}\left(M_{0}^{d}\right)$ is a sphere.

For the most part, this paper deals with the situation where $W(\lambda)=\sigma_{m}(\lambda)$ is the $m$ th elementary symmetric function of $\left(\lambda_{1}, \ldots, \lambda_{n}\right), 1<m<n$,

Received by the editors May 4, 1977 and, in revised form, June 27, 1977.

AMS (MOS) subject classifications (1970). Primary 53C45; Secondary 53C40.

${ }^{1}$ This research was supported in part by NSF Grant No. MPS 75-15733.

(C) American Mathematical Society 1979 
$\sigma_{m}(\lambda)=\Sigma \cdots \sum \lambda_{i(1)} \lambda_{i(2)} \cdots \lambda_{i(m)}, \quad$ where $i(1)<\cdots<i(m)$.

$H(x)=\sigma_{1}(\lambda(x)) / n$ is the mean curvature, $R(x)=2 !(n-2) ! \sigma_{2}(\lambda(x)) / n !$ the scalar curvature, $K(x)=\sigma_{n}(\lambda(x))$ the Gauss-Kronecker curvature; and more generally, $H_{m}(x)=m !(n-m) ! \sigma_{m}(\lambda(x)) / n !$ the $m$ th mean curvature $(1<m$ $\leqslant n$ ). Let $X(M)$ be a $\sigma_{m}$-Weingarten hypersurface, with $\sigma_{m}(\lambda(x)) \equiv C_{0}>0$. It is known that (b) holds if $m=1$ ([5]; cf. [12] for $n=2$ and [7] for a related result) or if $m=2$ ([6]). The object of this note is to prove:

TheOREM (*). Let $M=M^{n}$ be a complete connected Riemann manifold of class $C^{2}$ with nonnegative sectional curvature. Let $X: M \rightarrow R^{n+1}$ be an isometric immersion of class $C^{2}$ such that $X(M)$ has a positive constant mith mean curvature $H_{m}(x) \equiv C_{0}>0, x \in M$, for some $m, 1<m<n$. Then, in the Sacksteder decomposition (1.1)-(1.2), $X_{0}\left(M_{0}^{d}\right)$ is a sphere (of dimension $d$, $m<d<n)$.

We might remark that since $M$ and $X$ are of class $C^{2}$, they are real analytic, for $\sigma_{m}(\lambda(x)) \equiv C_{0}>0$ is a nonlinear elliptic analytic partial differential equation.

See [13] for the situation when $C_{m}=0$.

In $\$ \S 2$ and 3, we adapt the arguments used by Cheng and Yau [6] in the case $m=2$ to show that $X_{0}\left(M_{0}^{d}\right)$ is compact for arbitrary $m, 1<m<n$. Our proof depends on a generalization of the elliptic operator used in [21] and [6]. It then follows from results of Alexandrov [2] that $X_{0}\left(M_{0}^{d}\right)$ is a sphere; cf. Proposition 1.1 and remarks below. A different proof of the result in the compact case has been given by Nakagawa and Yokote [13, p. 479]. It should be noted that it has also been shown by Süss [17], Hsiung [11], U. Simon [16] and Yano [19] that when $M_{0}^{d}$ is compact with nonnegative sectional curvature and $X_{0}\left(M_{0}^{d}\right)$ is a $\sigma_{m}$-Weingarten hypersurface with $\sigma_{m}(\lambda(x)) \equiv C_{0}>0$ on $M$, and certain additional convexity properties are satisfied, then $X_{0}\left(M_{0}^{d}\right)$ is a sphere. By virtue of Sacksteder [15], Proposition 3.5 and its proof below, the additional convexity properties in Hsiung [11] and in U. Simon [16] are redundant and, in fact, Nakagawa and Yokote deduce their result from Hsiung's.

Actually, the fact that $X_{0}\left(M_{0}^{d}\right)$ is a sphere can be deduced from the following consequence (applied to $W=\sigma_{m}(\lambda)$ and $c>0$ ) of results of A. D. Alexandrov [2]:

Proposition 1.1. Let $W(\lambda)=W\left(\lambda_{1}, \ldots, \lambda_{n}\right)$ be of class $C^{1}$ for $0<\lambda_{1}$ $<\cdots<\lambda_{n}$ such that $\partial W / \partial \lambda_{k}>0$ for $1<k<n$. Let $M=M^{n}$ be $a$ compact connected Riemann manifold of class $C^{2}$ with nonnegative sectional curvature. Let $X: M \rightarrow R^{n+1}$ be an isometric immersion of class $C^{2}$ such that $X(M)$ is a W-Weingarten hypersurface, say, with $W(\lambda(x)) \equiv c$ on $M$ and that 


$$
\partial W(\lambda) / \partial \lambda_{k}>0 \text { for } 1<k<n
$$

whenever $\lambda \equiv \lambda(x), x \in M$ (for example, let (1.4) hold on the set $\{\lambda: W(\lambda)=$ $c\})$. Then $X(M)$ is a sphere.

Self-intersections are not excluded a priori in this assertion, but it follows from Sacksteder's results [15] that, in fact, $X(M)$ is the boundary of a convex body, so that $X$ is an embedding when $M$ and $X$ are sufficiently smooth.

Because A. D. Alexandrov strived for extreme generality in the statements of his theorems, some do not seem to be clearly worded; cf. the comments in [13, pp. 479-480] and in a footnote of the translator of [2, III, p. 391]. Nevertheless, it is not difficult to see that the proof of Proposition 1.1 is contained in Alexandrov's arguments in the proof of the result of $[2, \mathrm{~V}]$, with due reference to [2, III, pp. 390-391], and of course to [2, II, pp. 361-375]. (For an argument similar to [2, II, p. 371], in the special case $W=\sigma_{m}(\lambda)$, see [1, p. 827].)

Proposition 1.1 applies not only to $W(\lambda)=\sigma_{m}(\lambda)$ and $c>0$ (cf. Proposition 3.5 below) but, for example, also to $W(\lambda)=S_{1}^{m}-S_{m}$ and $c>0$, where $S_{m}=\lambda_{1}^{m}+\cdots+\lambda_{n}^{m}$ and $m=1,2, \ldots$ (and $W$ reduces to $S_{1}^{2}-S_{2}$ $=2 \sigma_{2}$ for $m=2$ ). Also, Proposition 1.1 and the proof of Theorem (*) have the following consequence:

COROLlaRY (*). Let $a_{1}, \ldots, a_{n}$ be nonnegative constants and

$$
W(\lambda)=\sum_{\mu=1}^{n} a_{\mu} \sigma_{\mu}(\lambda)
$$

Let $M=M^{n}$ be a complete Riemann manifold of class $C^{2}$ with nonnegative sectional curvatures. Let $X: M \rightarrow R^{n+1}$ be an isometric immersion of class $C^{2}$ such that $X(M)$ is a $W$-Weingarten hypersurface with $W(\lambda(x)) \equiv c>0$. Then, in the Sacksteder decomposition (1.1), $X_{0}\left(M_{0}^{d}\right)$ is a sphere (of dimension d, $0<d<n$ ).

ADDED IN PROOF (6/20/78). Using different methods in a forthcoming paper, we shall show that Theorem (*) remains valid if " $H_{m}(x) \equiv C_{0}$ " is replaced by " $W(\lambda(x)) \equiv c$ " where $W$ satisfies the conditions of Proposition 1.1.

2. Preliminaries for (a). As mentioned above, Cheng and Yau [6] show that a complete hypersurface in $R^{n+1}$ with nonnegative sectional curvatures and positive constant scalar curvature satisfies (b). From their arguments, we shall extract a general result (Lemma 2.1 below) dealing with the question of the validity of (a).

Unless otherwise indicated, all sums are over the range $1, \ldots, n$.

If $M$ is a Riemann manifold of class $C^{3}$, let $\omega_{1}, \ldots, \omega_{n}$ be a local $C^{2}$ orthonormal field of 1 -forms on $M$ satisfying the usual structure equations 


$$
\begin{aligned}
d \omega_{i} & =-\sum_{m} \omega_{i m} \omega_{m}, \quad \omega_{i m}+\omega_{m i}=0, \\
d \omega_{i j} & =-\sum_{m} \omega_{i m} \omega_{m j}+\Omega_{i j}, \\
\Omega_{i j} & =\frac{1}{2} \sum_{k} \sum_{m} R_{i j k m} \omega_{k} \omega_{m}, \quad R_{i j k m}+R_{i j m k}=0 .
\end{aligned}
$$

Here and below, products of exterior forms are understood to be exterior products. If a function $f$ is of class $C^{2}(M)$, its gradient or covariant derivative is defined by

$$
d f=\sum_{m} f_{, m} \omega_{m}
$$

and its second covariant derivative by

$$
\sum_{m} f_{, i m} \omega_{m}=d f_{, i}-\sum_{m} f_{, m} \omega_{m i} \text {. }
$$

Similarly, if $\Sigma \Sigma h_{i j} \omega_{i} \otimes \omega_{j}$ is a $C^{2}$ symmetric tensor on $M$, its first and second covariant derivatives are defined by

$$
\begin{aligned}
\sum_{m} h_{i j, m} \omega_{m} & =d h_{i j}-\sum_{m} h_{m j} \omega_{m i}-\sum_{m} h_{i m} \omega_{m j}, \\
\sum_{m} h_{i j, m k} \omega_{m} & =d h_{i j, k}-\sum_{m} h_{i j, m} \omega_{m k}-\sum_{m} h_{i m, k} \omega_{m j}-\sum_{m} h_{m j, k} \omega_{m i} \cdot
\end{aligned}
$$

Correspondingly, the Laplacian of the function $f$ and of the tensor $\Sigma \Sigma h_{i j} \omega_{i} \otimes$ $\omega_{j}$ are defined by

$$
\Delta f=\sum_{k} f_{, k k} \text { and } \Delta h_{i j}=\sum_{k} h_{i j, k k} \text {. }
$$

For any continuous symmetric tensor $\Sigma \Sigma \phi_{i j} \omega_{i} \otimes \omega_{j}$, define the differential operator $L_{\phi}$ of second order by

$$
L_{\phi} f=\sum_{1} \sum_{j} \phi_{i j} f_{, i j}
$$

This operator is called elliptic if $\left(\phi_{i j}\right)$ is positive definite, and degenerate elliptic if $\left(\phi_{i j}\right)$ is nonnegative definite.

Proposition 2.1 [6]. Let $M \in C^{3}$ and $\phi \in C^{1}$. Then $L_{\phi}$ is formally selfadjoint if and only if

$$
\sum_{j} \phi_{i j j} \equiv 0 \quad \text { for } 1<i<\text { non } M .
$$

A form of the maximum principle gives

Proposition 2.2 [5], [6. Let $L_{\phi}$ be formally selfadjoint and elliptic (possibly degenerate). Let $E \subset M$ be open with compact closure $\bar{E}, 0<f \in C^{2}(\bar{E})$, 
$0<g \in C^{2}(\bar{E}), g \neq 0$, and $g=0$ on $\partial E$. Then

$$
-\int_{E} g\left(L_{\phi} g\right) d x / \int_{E} g^{2} d x>\inf _{E}\left[-\left(L_{\phi} f\right) / f\right] .
$$

It will be convenient to list here, for ready reference, some assumptions which we will use from time to time in this and the following section.

(H1) $M=M^{n}$ is a complete connected Riemann manifold of class $C^{4}$ of nonnegative sectional curvature.

(H2) $X: M \rightarrow R^{n+1}$ is an isometric immersion of class $C^{4}$ such that $X(M)$ contains no complete lines, and so is the boundary of a convex body (for otherwise, we can replace $M, X$ by $M_{0}^{d}, X_{0}$ in the Sacksteder decomposition).

(H3) $X(M)$ is not compact.

(H4) $W=W(\lambda)$ is a $C^{2}$ function $\Phi$ of $S_{1}, \ldots, S_{m}$ for some $m>1$, where $S_{\mu}=\lambda_{1}^{\mu}+\cdots+\lambda_{n}^{\mu}$, i.e., $W(\lambda)=\Phi\left(S_{1}, \ldots, S_{m}\right)$.

Under the assumptions (H1)-(H2), there is a unit normal vector field $N$ : $M \rightarrow S^{n+1}$ such that the second fundamental form $\Sigma \Sigma h_{i j} \omega_{i} \otimes \omega_{j}$ is nonnegative definite. We, of course, have the Codazzi relations

$$
h_{i j, k}=h_{j i, k}=h_{i k, j}=h_{j k, i} \text {. }
$$

When (H3) holds, the normal image $N(M)$ is contained in a hemisphere, so that there exists a constant vector $U \in R^{n+1}$ such that the Euclidean scalar product

$$
N(x) \cdot U \geqslant 0 \text { on } M \text {; }
$$

Sacksteder (cf., e.g., [18]). If $\left\{e_{i}, \ldots, e_{n}\right\}$ is a local orthonormal frame field on $X(M)$, then the Gauss and Weingarten formulas are

$$
X_{, i j}=h_{i j} N \text { and } N_{, i}=-\sum_{j} h_{i j} e_{j}
$$

so that

$$
N_{, i j}=-\sum_{k} h_{i k} h_{k j} N-\sum_{k} h_{k i, j} e_{k}
$$

and consequently

$$
\begin{gathered}
L_{\phi} X=\sum_{i} \sum_{j} \phi_{i j} h_{j i} N \quad \text { or } \quad L_{\phi}(X \cdot U)=\sum_{i} \sum_{j} \phi_{i j} h_{j i}(N \cdot U), \\
L_{\phi}(N \cdot U)=-\sum_{i} \sum_{j} \sum_{k} \phi_{i j} h_{j k} h_{k i}(N \cdot U)-\sum_{i} \sum_{j} \sum_{k} \phi_{i j} h_{j i, k}\left(e_{k} \cdot U\right) ;
\end{gathered}
$$

cf. [7, p. 83].

Proposition 2.3. Assume (H1)-(H3) and (2.6). Let $L_{\phi}$ be elliptic and satisfy

$$
\sum_{i} \sum_{j} \phi_{i j} h_{j i, k} \equiv 0 \text { for } 1<k<n \text { on } M \text {. }
$$


Then $N(x) \cdot U>0$ on $M$

Proof. Even if $L_{\phi}$ is degenerate elliptic, we have that

$$
\sum_{i} \sum_{j} \phi_{i j} h_{j k} h_{i k} \geqslant 0 \text { for } 1<k<n .
$$

Hence, by (2.6) and (2.9), $L_{\phi}(N \cdot U)<0$ on $M$. The strong minimum principle of E. Hopf [10] for elliptic operators implies that if $N \cdot U=0$ at some point of $M$, then $N \cdot U \equiv 0$ on $M$. But this would show that, for every $x^{0} \in M$, the line $X\left(x^{0}\right)+t U,-\infty<t<\infty$, is on $X(M)$, which contradicts (H2).

Proposition 2.4. Assume (H1)-(H3). Let $L_{\phi}$ be formally selfadjoint, elliptic, and let (2.9) hold. Let $E=E(r)=\{x \in M: X(x) \cdot U<r\}$ for $r>0$. Then

$$
4 \int_{E(r)} \sum_{i} \sum_{j} \phi_{i j} h_{j i} d x / r \operatorname{vol} E(r / 2)>\inf _{E(r)} \sum_{i} \sum_{j} \sum_{k} \phi_{i j} h_{j k} h_{k i} \text {. }
$$

Proof. If we let $g=r-X(x) \cdot U$ and $f=N(x) \cdot U>0$, then Proposition 2.2 gives

$$
\begin{gathered}
\int_{E(r)}(r-X(x) \cdot U) \sum_{i} \sum_{j} \phi_{i j} h_{j i}(N \cdot U) d x / \int_{E(r)}(r-X(x) \cdot U)^{2} d x \\
>\inf _{E(r)} \sum_{i} \sum_{j} \sum_{k} \phi_{i j} h_{j k} h_{k i} .
\end{gathered}
$$

Since the left side of this relation is at most the left side of (2.10), the assertion follows.

Corollary 2.1. If, in addition to the assumptions of Proposition 2.4, we assume that

$$
\int_{E(r)} \sum_{i} \sum_{j} \phi_{i j} h_{j i} d x / r \text { vol } E(r / 2) \rightarrow 0 \text { as } r \rightarrow \infty,
$$

e.g., that

$$
\sum_{i} \sum_{j} \phi_{i j} h_{j i} \text { is bounded on } M \text {, }
$$

then

$$
\inf _{M} \sum_{i} \sum_{j} \sum_{k} \phi_{i j} h_{j k} h_{k i}=0
$$

Thus the arguments above lead to the following general result.

LeMma 2.1. Assume (H1)-(H2), and that (2.11) (say, (2.12)) holds but that (2.13) does not. Also, assume that $L_{\phi}$ is elliptic, formally selfadjoint, and that 
(2.9) holds. Then $X(M)$ is compact, i.e., (H3) cannot hold.

It will be seen, in the next section, that the assumption that " $L_{\phi}$ is formally selfadjoint" is quite restrictive.

3. Compactness. The object of this section is to prove the following:

LEMMA 3.1. Let $m$ be fixed, $1<m<n$. Assume conditions (H1)-(H2) of $\$ 2$ and that $X(M)$ is a $\sigma_{m}$-Weingarten hypersurface with $\sigma_{m}(\lambda(x))=C_{0}>0$ on $M$. Then $X(M)$ is compact.

This is a consequence of Lemma 2.1 and Propositions 3.1 and 3.4-3.7 below. In this section, we assume $(\mathrm{H} 1)-(\mathrm{H} 2)$ and $(\mathrm{H} 4)$. We associate with $W$, the symmetric tensor $\phi$ or $\Sigma \Sigma \phi_{i j} \omega_{i} \otimes \omega_{j}$ defined by

$$
\phi=\sum_{\mu=1}^{m} \mu\left(\partial W / \partial S_{\mu}\right) h^{\mu-1},
$$

where $h^{\mu}=\left(h_{i j}\right)^{\mu}$ is the $\mu$ th power of $h=\left(h_{i j}\right)$, the second fundamental matrix. Thus $h^{0}=I$ is the identity matrix and the $i j$ th element of $h_{i j}^{\mu}$ is

$$
h_{i j}^{\mu}=\sum_{i(1)} \cdots \sum_{i(\mu-1)} h_{i i(1)} h_{i(1) i(2)} \cdots h_{i(\mu-1) j} \text {. }
$$

Hence (3.1) means that

$$
\phi_{i j}=\sum_{\mu=1}^{m} \mu\left(\partial W / \partial S_{\mu}\right) h_{i j}^{\mu-1}, \quad \text { where } h_{i j}^{0}=\delta_{i j}
$$

Correspondingly, the associated operator is

$$
L_{\phi} f=\sum_{i} \sum_{j} \phi_{i j} f_{, i j}=\sum_{i} \sum_{j} \sum_{\mu=1}^{m} \mu\left(\partial W / \partial S_{\mu}\right) h_{i j}^{\mu-1} f_{, i j}
$$

Proposition 3.1. Under the assumptions (H1)-(H2), $(\mathrm{H} 4)$ and $W(\lambda(x)) \equiv$ const on $M$, the relations (2.9) hold.

Proof. Let $s_{\mu}(x)=S_{\mu}(\lambda(x))$. Then

$$
s_{\mu}(x)=\operatorname{tr} h^{\mu}=\sum_{i} \sum_{i(1)} \cdots \sum_{i(\mu-1)} h_{i i(1)} h_{i(1) i(2)} \cdots h_{i(\mu-1) i} .
$$

Hence, we have, with $i=i(\mu)$,

$$
\begin{aligned}
& s_{\mu, k}=\sum_{\kappa=1}^{\mu} \sum_{i} \sum_{i(1)} \cdots \sum_{i(\mu-1)} h_{i i(1)} \cdots h_{i(\kappa-1) i(\kappa), k} \\
& \cdot h_{i(\kappa) i(\alpha+1)} \cdots h_{i(\mu-1) i}
\end{aligned}
$$

so that

$$
s_{\mu, k}=\mu \operatorname{tr}\left(h^{\mu-1} h_{, k}\right)=\mu \sum_{i} \sum_{j} h_{i j}^{\mu-1} h_{j i, k}
$$


Note that if $w(x)=W(\lambda(x))$, then $w(x) \equiv c_{0}$ on $M$ implies that

$$
0=w_{, k}=\sum_{\mu=1}^{m}\left(\partial W / \partial S_{\mu}\right) s_{\mu, k}=\sum_{i} \sum_{j} \phi_{i j} h_{j i, k} \text { for } 1<k<n .
$$

Proposition 3.2. Assume (H1)-(H2), (H4) and (3.1). Then $L_{\phi}$ is elliptic if and only if

$$
\partial W(\lambda) / \partial \lambda_{i}>0 \text { for } \lambda=\lambda(x), x \in M, 1<i<n .
$$

PRoof. In fact, the eigenvalues of $\phi$ are

$$
\sum_{\mu=1}^{m} \mu\left(\partial W / \partial S_{\mu}\right) \lambda_{i}^{\mu-1}=\partial W / \partial \lambda_{i} \quad \text { with } \lambda=\lambda(x), 1<i<n .
$$

Proposition 3.3. Under the assumptions (H1)-(H2), (H4) and (3.1), a sufficient condition for $L_{\phi}$ to be formally selfadjoint is that

$$
\begin{aligned}
\sum_{\kappa=1}^{m} \sum_{\mu=1}^{m} \mu \kappa\left(\partial^{2} W / \partial S_{\kappa} \partial S_{\mu}\right) \lambda_{i}^{\mu-1} \lambda_{k}^{\kappa-1} \\
\quad+\sum_{\kappa=1}^{m-1} \sum_{\mu=\kappa+1}^{m} \mu\left(\partial W / \partial S_{\mu}\right) \lambda_{i}^{\mu-\kappa-1} \lambda_{k}^{\kappa-1}=0
\end{aligned}
$$

for $1 \leqslant i, k \leq n$. This is the case, e.g., if $W$ satisfies

$$
\kappa \sum_{\mu=1}^{m} \mu\left(\partial^{2} W / \partial S_{\kappa} \partial S_{\mu}\right) \lambda_{i}^{\mu-1}+\sum_{\mu=\kappa+1}^{m} \mu\left(\partial W / \partial S_{\mu}\right) \lambda_{i}^{\mu-\kappa-1}=0
$$

for $1<i<n, 1 \leqslant \kappa<m$.

Proof. From (3.3),

$$
\sum_{j} \phi_{i j, j}=\sum_{j}\left\{\sum_{\mu=1}^{m} \sum_{k=1}^{m} \mu\left(\partial^{2} W / \partial S_{k} \partial S_{\mu}\right) s_{\kappa, j} h_{i j}^{\mu-1}+\sum_{\mu=2}^{m} \mu\left(\partial W / \partial S_{\mu}\right) h_{i j j}^{\mu-1}\right\} \text {. }
$$

Note that (3.2) implies that

$$
h_{i j, j}^{\mu-1}=\sum_{\kappa=1}^{\mu-1} \sum_{k} \sum_{p} h_{i k}^{\kappa-1} h_{k p j} h_{p j}^{\mu-\kappa-1} .
$$

By the Codazzi relations (2.5), we have $h_{k p j}=h_{j p, k}$, so that, by (3.6),

$$
\begin{aligned}
\sum_{j} h_{i j j}^{\mu-1} & =\sum_{\kappa=1}^{\mu-1}(\mu-\kappa)^{-1} \sum_{k} h_{i k}^{\kappa-1} s_{\mu-\kappa, k} \\
& =\sum_{\kappa=1}^{\mu-1} \kappa^{-1} \sum_{j} h_{i j}^{\mu-\kappa-1} s_{\kappa, j}
\end{aligned}
$$

for $\mu>2$. If, at a fixed arbitrary point $x$ of $M, h=\operatorname{diag}\left(\lambda_{1}(x), \ldots, \lambda_{n}(x)\right)$, 
then, by (3.6) and the last relation,

$$
\begin{aligned}
s_{\kappa, j} & =\kappa \sum_{k} \lambda_{k}^{\kappa-1} h_{k k, j}, \\
\sum_{j} h_{i j, j}^{\mu} & =\sum_{\kappa=1}^{\mu-1} \kappa^{-1} \lambda_{i}^{\mu-\kappa-1} S_{\kappa, i}=\sum_{\kappa=1}^{\mu-1} \sum_{k} \lambda_{i}^{\mu-\kappa-1} \lambda_{k}^{\kappa-1} h_{k k, i} .
\end{aligned}
$$

Hence, $\Sigma_{j} \phi_{i j, j}$ becomes

$$
\begin{aligned}
\sum_{k}\left\{\sum_{\kappa=1}^{m} \sum_{\mu=1}^{m} \mu \kappa\right. & \left(\partial^{2} W / \partial S_{\kappa} \partial S_{\mu}\right) \lambda_{i}^{\mu-1} \lambda_{k}^{\kappa-1} \\
& \left.+\sum_{\mu=2}^{m} \sum_{\kappa=1}^{\mu-1} \mu\left(\partial W / \partial S_{\mu}\right) \lambda_{i}^{\mu-\kappa-1} \lambda_{k}^{\kappa-1}\right\} h_{k k, i} .
\end{aligned}
$$

Thus, the first part of the assertion follows, and the second is obvious (since $\partial W / \partial S_{m+1}=0$ ).

Proposition 3.4. If $W=\sigma_{m}(\lambda)$, then (3.10) holds. Hence if (H1)-(H2) holds and $W=\sigma_{m}(\lambda)$ in (3.1), then $L_{\phi}$ is selfadjoint.

It is not difficult to see that if $W(\lambda)$ is a symmetric homogeneous polynomial of degree $m$ in $\left(\lambda_{1}, \ldots, \lambda_{n}\right)$, then (3.10) holds only if $W$ is a constant multiple of $\sigma_{m}$.

Proof. Standard formulas show that

$$
\mu \partial \sigma_{m} / \partial S_{\mu}=(-1)^{\mu-1} \sigma_{m-\mu} \text { for } 1<\mu<m ;
$$

cf. $[4, \S 80$ or 161$]$, where $p_{\mu}=(-1)^{\mu} \sigma_{\mu}$. Hence $\mu \kappa \partial^{2} \sigma_{m} / \partial S_{\kappa} \partial S_{\mu}=(-1)^{\mu+\kappa} \sigma_{m-\mu-\kappa}$ for $1<\kappa, \mu<m-1$, and $\mu+\kappa<m$.

Thus, if $W=\sigma_{m}$, then the left side of (3.10) is

$$
\sum_{\mu=1}^{m-\kappa}(-1)^{\mu+\kappa} \sigma_{m-\mu-\kappa} \lambda_{i}^{\mu-1}-\sum_{\mu=\kappa+1}^{m}(-1)^{\mu} \sigma_{m-\mu} \lambda_{i}^{\mu-\kappa-1}=0 .
$$

Proposition 3.5. If $0<\lambda_{1}^{0}<\cdots<\lambda_{n}^{0}$ and $\sigma_{m}\left(\lambda^{9}\right)>0$, then $\partial \sigma_{m}\left(\lambda^{9}\right) / \partial \lambda_{k}$ $>0$ for $1<k<n$. Hence, if (H1)-(H2) holds, $W=\sigma_{m}(\lambda)$ in (3.1), and $\sigma_{m}(\lambda(x))>0$ on $M$, then $L_{\phi}$ is elliptic.

Proof. Note that

$$
\partial \sigma_{m}(\lambda) / \partial \lambda_{i}=\sigma_{m-1}^{(i)}(\lambda),
$$

where $\sigma_{m-1}^{(i)}$ denotes the $(m-1)$ st elementary symmetric function in $\lambda_{1}, \ldots, \lambda_{i+1}, \lambda_{i+1}, \ldots, \lambda_{n}$. We shall first verify the following: 
Claim. Let $0 \leqslant \lambda_{1}^{0}<\cdots<\lambda_{n}^{0}$. Then $\sigma_{m}\left(\lambda^{g}\right)=0$ if and only if there exist $m-1$ indices $(1 \leqslant) i(1)<\cdots<i(m-1)(<n)$ such that $\lambda_{k}^{0}=0$ if $k \neq$ $i(\mu), 1<\mu<m-1$.

This is clear if $m=1$. Assume that $m>1$ and that the claim holds if $m$ is replaced by $m-1$. Suppose that $\lambda_{j}^{0} \neq 0$ for some $j$. Since $m \sigma_{m}(\lambda)=$ $\Sigma \lambda_{i} \sigma_{m-1}^{(i)}(\lambda), \sigma_{m}\left(\lambda^{9}\right)=0$ implies that $\sigma_{m-1}^{(j)}\left(\lambda^{9}\right)=0$. Thus, by the induction hypothesis, there exist $m-2$ indices $(1<) i(1)<\cdots<i(m-2)(<n)$, $i(\mu) \neq j$, such that $\lambda_{k}^{0}=0$ if $k \neq j$ or $k \neq i(\mu), 1<\mu<m-2$. This gives the claim.

COMPLETION OF THE PROOF. Suppose that $0<\lambda_{1}^{0}<\cdots<\lambda_{n}^{0}$ and that $\partial \sigma_{m}\left(\lambda^{0}\right) / \partial \lambda_{i}=0$ for some $i, 1<i<n$. Then $\sigma_{m-1}^{(i)}\left(\lambda^{9}\right)=0$, and so there exist $m-2$ indices $i(1)<\cdots<i(m-2), i(\mu) \neq i$, such that $\lambda_{j}^{0}=0$ if $j \neq i$ or $j \neq i(\mu)$ for $1<\mu<m-2$. Thus $\sigma_{m}\left(\lambda^{9}\right)=0$. This proves the first part of the proposition, and the other part follows from Proposition 3.2.

REMARK. It is clear that this proof shows that if $c_{0}>0$ and $c_{1}>0$, then there exists a $\delta=\delta\left(c_{0}, c_{1}\right)>0$ such that

$$
\partial \sigma_{m}(\lambda) / \partial \lambda_{k}>\delta>0 \text { if } \sigma_{m}(\lambda)>c_{0}>0,0<\lambda_{1}<\cdots<\lambda_{n}<c_{1} .
$$

Proposition 3.6. Assume (H1)-(H2), (H4) and that $W(\lambda)$ is a homogeneous function of degree $m$ in (3.1). Then

$$
\operatorname{tr}(\phi h)=\sum_{i} \sum_{j} \phi_{i j} h_{j i}=m W(\lambda(x)),
$$

so that (2.12) holds if $W(\lambda(x)) \equiv$ const on $M$.

Proof. By (3.8), the eigenvalues of $\phi h$ are

$$
\sum_{\mu=1}^{m} \mu\left(\partial W / \partial S_{\mu}\right) \lambda_{i}^{\mu}=\lambda_{i} \partial W / \partial \lambda_{i}, \quad 1<i<n,
$$

so that (3.16) follows from Euler's theorem.

Proposition 3.7. If (H1)-(H2) holds, $W=\sigma_{m}(\lambda)$ in (3.1), and $\sigma_{m}(\lambda(x))>$ $C_{0}>0$ on $M$, then

$$
\inf _{M} \operatorname{tr}\left(\phi h^{2}\right)=\inf _{M} \sum_{i} \sum_{j} \sum_{k} \phi_{i j} h_{j k} h_{k i}>0 .
$$

Proof. The eigenvalues of $\phi h^{2}$ are

$$
\sum_{\mu=1}^{m} \mu\left(\partial W / \partial S_{\mu}\right) \lambda_{i}^{\mu+1} \text { for } 1<i<n,
$$

so that

$$
\operatorname{tr}\left(\phi h^{2}\right)=\sum_{\mu=1}^{m} \mu\left(\partial W / \partial S_{\mu}\right) S_{\mu+1}
$$


When $W=\sigma_{m},(3.12)$ implies that

$$
\operatorname{tr}\left(\phi h^{2}\right)=\sum_{\mu=1}^{m}(-1)^{\mu-1} \sigma_{m-\mu} S_{\mu+1}=\sum_{\mu=2}^{m+1}(-1)^{\mu} \sigma_{m+1-\mu} S_{\mu} .
$$

Thus, by Newton's formula (cf. [3, p. 244]),

$$
\operatorname{tr}\left(\phi h^{2}\right)=\sigma_{m} \sigma_{1}-(m+1) \sigma_{m+1},
$$

where $\sigma_{m+1}=0$ if $m=n$. If $m<n$, then each term $\lambda_{i(1)} \cdots \lambda_{i(m+1)}$ of $\sigma_{m+1}$ occurs $m+1$ times in $\sigma_{m} \sigma_{1}$, for the factor $\lambda_{i(j)}$ can be considered to be in $\sigma_{1}$ for $j=1, \ldots, m+1$. Thus

$$
\operatorname{tr}\left(\phi h^{2}\right) \geqslant \sum_{\mu=1}^{m}\left\{\Sigma \cdots \Sigma \lambda_{i(1)} \cdots \lambda_{i(\mu-1)} \lambda_{i(\mu)}^{2} \lambda_{i(\mu+1)} \cdots \lambda_{i(m)}\right\},
$$

where the inner sum is over $1<i(1)<\cdots<i(m)<m$. Hence

$$
\operatorname{tr}\left(\phi h^{2}\right) \geqslant \lambda_{n}\left(\lambda_{n} \lambda_{n-1} \cdots \lambda_{n-m+1}\right) .
$$

Since $\sigma_{m}<\left(\lambda_{n} \cdots \lambda_{n-m+1}\right) n ! /(n-m) ! m !<\lambda_{n}^{m} n ! /(n-m) ! m !$, we see that

$$
\operatorname{tr}\left(\phi h^{2}\right) \geqslant\left[m !(n-m) ! \sigma_{m} / n !\right]^{1+1 / m} \geqslant\left[m !(n-m) ! C_{0} / n !\right]^{1+1 / m}>0 .
$$

\section{REFERENCES}

1. A. Aeppli, On the uniqueness of compact solutions for certain elliptic differential equations, Proc. Amer. Math. Soc. 11 (1960), 826-832.

2. A. D. Alexandrov, Uniqueness theorems for surfaces in the large. II, III, and V, Vestnik Leningrad Univ. Math. 12 (1957), no. 7, 15-44; 13 (1958), no. 7, 14-26; and 13 (1958), no. 19, 5-8; Amer. Math. Soc. Transl. (2) 21 (1962), 354-388, 389-403 and 412-416.

3. M. Bôcher, Introduction to higher algebra, Macmillan, New York, 1933.

4. W. S. Burnside and A. W. Panton, The theory of equations, vols. I and II, reprint, Dover, New York, 1960.

5. S. Y. Cheng and S. T. Yau, Differential equations on Riemannian manifolds, Comm. Pure Appl. Math. 28 (1975), 333-354.

6. __ Hypersurfaces with constant scalar curvature, Math. Ann. 225 (1977), 195-204.

7. S. S. Chern, On the curvatures of a piece of hypersurface in Euclidean space, Abh. Math. Sem. Univ. Hamburg 29 (1965), 76-91.

8. P. Hartman, On the isometric immersions in Euclidean space of manifolds with nonnegative sectional curvatures. II, Trans. Amer. Math. Soc. 147 (1970), 529-540.

9. 37-48. , Some characterizations of the Euclidean sphere, Nonlinear Analysis: TMA 1 (1977),

10. E. Hopf, Elementare Bemerkungen über die Lösungen partieller Differentialgleichungen Zweiter Ordnung vom Elliptischen Typus, Sitzungsber. Preuss. Akad. Wiss. 19 (1927), 147-152.

11. C. C. Hsiung, Some integral formulas for clased hypersurfaces, Math. Scand. 2 (1959), 286-294.

12. T. Klotz and R. Osserman, Complete hypersurfaces in $E^{3}$ with constant mean curvature, Comment. Math. Helv. 41 (1966/67), 313-318.

13. H. Nakagawa and I. Yokote, On hypersurfaces with constant scalar curvature in a Riemann manifold of constant curvature, Kōdai Math. Sem. Rep. 24 (1972), 471-481.

14. K. Nomizu and B. Smyth, A formula of Simons' type and hypersurfaces with constant mean curvature, J. Differential Geometry 3 (1969), 367-377. 
15. R. Sacksteder, On hypersurfaces with no negative sectional curvatures, Amer. J. Math. 82 (1960), 609-630.

16. U. Simon, Minkowskische Integralformen und ihre Anwendungen in der Differentialgeometrie im Grossen, Math. Ann. 173 (1967), 307-321.

17. W. Süss, Zur relative Differentialgeometrie. V. Über Eiflächen in $R^{n+1}$, Tôhoku Math. J. 31 (1929), 202-209.

18. H. Wu, The spherical images of convex hypersurfaces, J. Differential Geometry 9 (1974), 279-290.

19. K. Yano, Integral formulas in Riemannian geometry, Marcel Dekker, New York, 1970.

20. S. T. Yau, Submanifolds with constant mean curvature. I, Amer. J. Math. 96 (1974), 346-366. 21. , Hypersurfaces with constant scalar curvature, Preprint.

Mathematics Department, The Johns Hopkins University, Baltimore, Maryland 21218 